\title{
Chronic Intermittent Hypobaric Hypoxia Prevents contrast-Induced Acute Kidney Injury by Modulating the HIF-1a Signaling Pathway
}

\section{Kai-min Yin}

Graduate School of Hebei North University

\section{Yan-hui Ni}

Hebei General Hospital

\section{Guang-yun Cao}

Hebei General Hospital

Jia-yuan Zhang

Graduate School of Hebei North University

\section{Bao-jun Yi}

Graduate School of Hebei North University

\section{Zi-hao Pang}

Graduate School of Hebei North University

\section{Hui-jie Ma}

Hebei Medical University

Li Zhang ( $1136329347 @ q q . c o m)$

Hebei General Hospital

\section{Research Article}

Keywords:

Posted Date: February 15th, 2022

DOI: https://doi.org/10.21203/rs.3.rs-1308222/v1

License: (1) (1) This work is licensed under a Creative Commons Attribution 4.0 International License. Read Full License 


\section{Abstract}

The aim of this study was to explore the role of $\mathrm{ClHH}$ in preventing contrast-induced acute kidney injury (Cl-AKI) in rats and its mechanism. Rats mean arterial pressure, heart rate, blood creatinine and urea nitrogen levels were measured. The kidney tissue pathological changes, superoxide dismutase (SOD) activity, malondialdehyde (MDA) levels, HIF-1 $\mathrm{a} \square \mathrm{Bcl}-2$ 19-kDa interacting protein3 (BNIP3) $\square$ caspase3and poly(ADP-ribose) polymerase (PARP) expression levels were testing. The results showed that $\mathrm{ClHH}$ pretreatment $\mathrm{Cl}-\mathrm{AKI}$ group mean arterial pressurenheart ratelblood creatinine and urea nitrogen levels were reduced, kidney tissue SOD activity was increased, MDA levels was reduced, HIF-1a囚 BNIP3■ caspase 3 and PARP levels were increased than the $\mathrm{Cl}-\mathrm{AKI}$ group. This study indicates that $\mathrm{ClHH}$ pretreatment may have a protective effect on contrast-induced early kidney injury by activating the HIF1a/BNIP3 signaling pathway to regulate mitochondrial autophagy and enhance cellular anti-apoptotic and renal antioxidant capacity, for the first time.

\section{Introduction.}

Although the incidence of $\mathrm{Cl}-\mathrm{AKI}$ has been reduced by the application of low osmotic / isotonic nonionic contrast agents, the incidence rate of $\mathrm{Cl}-\mathrm{AKI}$ is increasing due to the increase in high-risk groups such as elderly patients, diabetes mellitus and chronic kidney disease. It has been reported that $\mathrm{Cl}-\mathrm{AKI}$ has become the third leading cause of medically induced acute kidney injury[1].Once contrast renal damage occurs, there is still no effective treatment. Therefore, it is particularly important to prevent the occurrence of $\mathrm{Cl}-\mathrm{AKI}$. The pathogenesis of $\mathrm{Cl}-\mathrm{AKI}$ is still unclear, but studies have shown that there are multiple potential pathways of interaction in the Cl-AKI happen and development, including ischemia, hypoxia and oxidative stress due to altered renal hemodynamics caused by vasoactive substances and the high viscosity of the contrast agent itself, and direct toxic effects of the contrast agent itself on renal tubular epithelial cells and endothelial cells[2].

SOD has the function of scavenging ROS, after receiving contrast agent, the kidney undergoes oxidative stress, the activity of SOD in serum and kidney tissue decreases, the level of ROS and MDA increases, and ROS can directly cause tubular cell damage[3].Under hypoxia, the body activates hypoxia inducible factor (HIF) to regulate the expression of various genes to adapt to the hypoxic environment, such as genes involved in cell survival, angiogenesis, glycolysis, and invasion/metastasis[4]. HIF consists of three main a subunits (HIF-1a, HIF-2a, HIF-3a) and one $\beta$ subunit (HIF-1 $\beta$ ), HIF-1a and HIF-2a will becoming the main regulatory factors produced under hypoxic conditions[5]. It was shown that the expression levels of HIF-1a and BNIP3 were increased in contrast-induced acute kidney injury, and further increase of both expression could enhance mitochondrial autophagy and attenuate apoptosis to improve contrast kidney injury[6-8]. Caspase-3 is the major terminal shear enzyme in apoptosis, and its main substrate is PARP. When the body was stimulated, caspase 3 was activated into cleaved-caspase3, cleaved-caspase 3 could activate PARP to induce apoptosis. Romano et al study shows, human embryonic kidney cells (HEK 293), porcine proximal renal tubular cells (LLC-PK1) and Madin-Darby renal epithelial cells (MDCK) were incubated with lodine contrast agent injections, caspase3 and RARP levels were reduced, cell activity was 
reduced, and apoptosis was increased, suggesting that iodine contrast agents can activate the caspase 3 pathway to cause apoptosis in kidney cells[9]. Studies mentioned above showed that modulation of HIF1a signaling pathway and inhibition of caspase-3 activation could enhance mitochondrial autophagy and attenuate apoptosis to protect the kidney. Thus, the changes of HIF-1a and caspase-3 levels were used as the main observation indicators in this experiment.

Current studies have shown the existence of multiple benefits of $\mathrm{ClHH}$ to the organism. For example, $\mathrm{ClHH}$ ameliorates renal injury in rats with diabetic nephropathy by activating the HIF1-a signaling pathway[10]; intermittent hypobaric hypoxia enhances HIF stability by inhibiting prolyl hydroxylase (PHD) activity, which activates key adaptive genes to attenuate hypoxic injury in the body[11] $\mathbb{C l H H}$ also has the ability to improve hypertension[12,13], improve left ventricular remodeling and myocardial fibrosis[14],anti-arrhythmia[15, 16], attenuate oxidative stress[17], alter cardiac energy metabolism[18], reduce the size of myocardial infarction[19], inhibit inflammatory response and apoptosis[20], improve insulin resistance[13], attenuate endoplasmic reticulum stress[21].

Therefore, we hypothesized that $\mathrm{CIHH}$ may protect against $\mathrm{Cl}-\mathrm{AKI}$ by modulating the HIF signaling pathway, inhibiting renal oxidative stress, and enhancing mitochondrial autophagy and cellular antiapoptotic capacity. In this study, we used histological and molecular biological methods to assess the protective effects of $\mathrm{ClHH}$ on $\mathrm{Cl}$-AKI rats induced by indomethacin, $\mathrm{N} \omega$-nitro-L-arginine methyl ester and ioversol injection, and investigate the mechanism of action.

\section{Materials And Methods.}

\subsection{Animals and $\mathrm{ClHH}$ pretreatment}

Male Sprague-Dawley (SD) rats, SPF grade, 24 in total (weight $160 \pm 10 \mathrm{~g}$, buy from Hebei Ivivo Biotechnology Co., Ltd.), were purchased and kept in the laboratory of Hebei Medical University, where the laboratory temperature was constant at $22 \pm 1^{\circ} \mathrm{C}$ and received light/dark time for $12 \mathrm{~h}$ each, with free access to water and food. At the beginning of the experiment, 24 male SD rats were randomly divided into 4 groups of 6 rats each: normal-controlled group (CTL, rats were kept in the laboratory environment at all times), Cl-AKI group (rats were kept in the laboratory environment at all times), $\mathrm{ClHH}$ group (SD rats were placed in a hypobaric hypoxic chamber simulating $4000 \mathrm{~m}$ altitude for $5 \mathrm{~h}$ per day for 35 days), $\mathrm{ClHH}$ prevented $\mathrm{Cl}-\mathrm{AKI}$ group ( SD rats were placed in a hypobaric hypoxic chamber simulating $4000 \mathrm{~m}$ altitude for 5 hours per day for 35 days before Cl-AKI modeling). The experiment was approved by the ethics committee of Hebei Provincial People's Hospital and completed by the Department of physiology of Hebei Medical University. The study was carried out in compliance with the ARRIVE guidelines.

\subsection{Cl-AKI rats model establishment}

The modeling method was referred to Bae et al.[22], as follows: rats in the $\mathrm{Cl}-\mathrm{AKI}$ group and $\mathrm{CIHH}$ to prevent Cl-AKI group were sequentially injected with indomethacin $(10 \mathrm{mg} / \mathrm{kg}$, Aladdin,China,1106885-5g, 


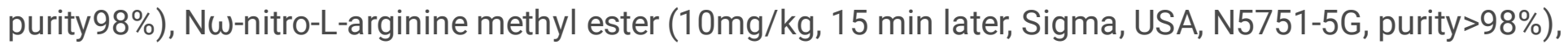
and ioversol injection $(8.3 \mathrm{~mL} / \mathrm{kg}, 30 \mathrm{~min}$ later $\ 350 \mathrm{mgl} / \mathrm{ml}$, Jiangsu Hengrui Pharmaceutical Co., Ltd, China) through the tail vein, and rats in the $\mathrm{CTL}$ and $\mathrm{ClHH}$ groups were injected with saline through the tail vein $0.5 \mathrm{~mL} /$ time, 3 times in total. After injection, all rats were kept in the laboratory environment and resumed free access to water and food.

\section{Detection methods of various indicators.}

\subsection{Heart rate and mean arterial pressure measurements}

Heart rate and mean arterial pressure were measured by a tail cuff manometer (LE5001, Panlab) every morning (7:00-10:00 am) from one week before injecting drug until 24 hours after contrast agent injection, or before blood collection if blood specimens were to be collected from the tail vein.

\subsection{Testing of biochemical indicators}

Blood was collected from the tail vein of rats before and 24 hours after contrast agent injection, respectively. Then centrifuged them for $15 \mathrm{~min}$ at $4^{\circ} \mathrm{C}$ and $3000 \mathrm{rpm}$ using a tabletop high-speed refrigerated centrifuge (DaLong, D3024R), and the supernatant was taken for the determination of serum creatinine and serum urea nitrogen by an automatic biochemical analyzer (Shenzhen Redu Life Science, Chemray800).

\subsection{Measurement of Biomarkers of Oxidative Stress}

The rats were killed by neck leading at $24 \mathrm{~h}$ after contrast agent injection, and the isolated right kidney tissues were washed with saline (pre-cooled on ice in advance) to remove residual blood and homogenized, then using a benchtop high speed frozen centrifuge (DaLong, D3024R) to centrifuge for 15 min at $4{ }^{\circ} \mathrm{C}, 3000 \mathrm{rpm} / \mathrm{min}$, and the supernatant was collected. SOD activity and MDA levels were determined by using two commercial experimental kits (Nanjing Jiancheng Institute of Biological Engineering) according to the assay instructions of the kits[23].

\subsection{Histological examination}

Rats were executed 24 hours after drug injection, and the left kidney was fixed in $4 \%$ paraformaldehyde solution for 48 hours and then dehydrated in gradient alcohol, transparent in xylene, and embedded in paraffin wax. The paraffin kidney tissue block was cut into thin slices of approximately $3 \mu \mathrm{m}$ thickness using a microtome (Leica, Germany), then stained with hematoxylin eosin staining (HE staining), periodic acid Schiff reaction (PAS)and Masson trichrome after dewaxing in xylene and rehydrating in gradient 
alcohol. Last observed and photographed in positive White light microscope (Nikon (Japan), Eclipse Ci-L), and each tissue section was carefully observed under the microscope and histological changes were recorded.

\subsection{Immunoblot analysis}

Rats were executed $24 \mathrm{~h}$ after drug injection, the right kidney was taken and cut with tissue scissors. The kidney tissues were homogenized in tissue/cell lysate. Protein samples were separated by SDS-PAGE, transferred to a PVDF membrane (Millipore Corporation, USA) that was blocked for $1 \mathrm{~h}$ with $5 \%(\mathrm{w} / \mathrm{v})$ nonfat milk in Tris-buffered saline, and incubated with antibodies against HIF-1a (1:500, Wanleibio, China, WL01607), BNIP3 (1:1500, Wanleibio, China, WL01139), caspase3 (1:1500, Affinity, USA, AF6311)), PARP (1:750, Wanleibio, China, WL01932), overnight at $4{ }^{\circ} \mathrm{C}$ on a constant temperature refrigerator shaker. The same membrane was stripped and re-blotted with an $\beta$-actin antibody (1:5000, Proteintech, USA, 20536-1AP) for normalization. Blots were developed by the chemiluminescent detection method (Amer sham $E C L)$. The protein blots were quantified by densitometry using Image $\mathrm{J}$ software and normalized to $\beta$ actin.

\subsection{Data analysis}

All data were performed with GraphPad Prism 9.0.0冈121囚software. All groups with $n=6$ each were analyzed by one-way analysis of variance (ANOVA). Results are presented as mean \pm SEM and $P<0.05$ was considered significant.

\section{Results}

\subsection{Effect of $\mathrm{ClHH}$ on Heart Rate and Mean Arterial Pressure}

One week before injection, all rats heart rate and mean arterial pressure were not difference. However, 24 hours after contrast agent injection, the heart rate and mean arterial pressure were significantly higher in the $\mathrm{Cl}-\mathrm{AKI}$ and $\mathrm{ClHH}$ prevented $\mathrm{Cl}-\mathrm{AKI}$ groups compared with the $\mathrm{CTL}$ group, and were significantly lower in the $\mathrm{ClHH}$ prevented $\mathrm{Cl}-\mathrm{AKI}$ group compared with the $\mathrm{Cl}-\mathrm{AKI}$ group $(\mathrm{P}<0.05$, Fig 1$)$.

\subsection{Effect of $\mathrm{ClHH}$ on renal function.}

Serum creatinine and blood urea nitrogen levels were significantly higher in the $\mathrm{Cl}-\mathrm{AKI}$ group and $\mathrm{ClHH}$ prevented $\mathrm{Cl}-\mathrm{AKI}$ group compared with the CTL group, but lower in the $\mathrm{ClHH}$ prevented $\mathrm{Cl}-\mathrm{AKI}$ group compared with the $\mathrm{Cl}-\mathrm{AKI}$ group $(\mathrm{P}<0.05$, Fig 2$)$. 


\subsection{Histological examination}

HE staining showed that the water like degeneration of renal tubular epithelial cells in Cl-AKI group was obvious, eosinophilic masses were seen in a large number of renal tubular lumens, some renal tubules were necrotic, and the lumen structure of renal tubules was not clear. There was a small amount of watery degeneration of renal tubular epithelial cells in $\mathrm{ClHH}$ prevented $\mathrm{Cl}$-AKI group, a small amount to medium amounts of eosinophilic masses in the lumen of renal tubules, and no tubular necrosis and changes in lumen structure were observed. PAS staining showed no obvious changes in glomerulus mesangial region, and Masson staining showed no obvious fibrosis changes in glomerulus and renal stroma in all groups (Fig 3).

\subsection{Antioxidant effects of $\mathrm{ClHH}$}

The activity of SOD was significantly decreased in CI-AKI group compared to CTL group 囚but was significantly increased in $\mathrm{CIHH}$ prevented $\mathrm{Cl}-\mathrm{AKI}$ group compared to $\mathrm{Cl}-\mathrm{AKI}$ group $(\mathrm{P}<0.05$, Fig. $4 \mathrm{a})$. In addition, the levels of MDA were significantly higher in Cl-AKI group compared to CTL group, but were significantly lower in $\mathrm{ClHH}$ prevented group compared to $\mathrm{Cl}-\mathrm{AKI}$ group $(\mathrm{P}<0.05$, Fig. $4 \mathrm{~b})$.

\subsection{Western Blot}

Our dates indicated that HIF-1a and BNIP3 protein expression levels were significantly increased in CI-AKI group compared to CTL group, and were more increased in $\mathrm{ClHH}$ prevented $\mathrm{Cl}-\mathrm{AKI}$ group compared to $\mathrm{Cl}-$ AKI group. The results also showed that caspase 3 and PARP levels were both down-regulated in $\mathrm{Cl}-\mathrm{AKI}$ group compared to $\mathrm{CTL}$ group, while increased inClHH prevented $\mathrm{Cl}-\mathrm{AKI}$ group compared to $\mathrm{Cl}-\mathrm{AKI}$ group $(P<0.05$, Fig. 5).

\section{Discussion}

The incidence of $\mathrm{Cl}-\mathrm{AKI}$ has decreased in recent years with the use of isotonic/hypotonic contrast agents, but the number of $\mathrm{Cl}-\mathrm{AKI}$ cases is still increasing. In the general population, the incidence of $\mathrm{Cl}-\mathrm{AKI}$ is $2.0 \%$, but in the population with comorbid chronic kidney disease, diabetes, advanced age, and emergency percutaneous coronary intervention (PCI), the incidence of $\mathrm{Cl}-\mathrm{AKI}$ rises to 30\%[24]. Although studies have shown that some drugs or methods have a preventive effect on $\mathrm{Cl}-\mathrm{AKI}$, such as lignans[25], acetylcysteine[26], sodium bicarbonate[27], hydration[28]etc. But, there is still no consensus on these methods. Once Cl-AKI occurs, there is no effective treatment. Therefore, it is particularly important to prevent the occurrence of $\mathrm{Cl}-\mathrm{AKI}$. CIHH has been shown to have many benefits to the organism both in the exercise business and in animal experiments. The present study is the first to suggest that $\mathrm{CIHH}$ 
pretreatment before administration of contrast media can prevent or attenuate the occurrence of $\mathrm{Cl}-\mathrm{AKI}$ in rats.

In the present study, the rats in the $\mathrm{Cl}-\mathrm{AKI}$ group showed increased heart rate, elevated mean arterial pressure, significantly increased blood creatinine and blood urea nitrogen, aqueous degeneration of renal tubular epithelial cells, eosinophilic masses in the lumen of a large number of renal tubules, tubular necrosis, and unclear tubular lumen structure, suggesting that the rats in the contrast nephropathy model had significant renal injury. Although the rats blood creatinine and blood urea nitrogen levels in the $\mathrm{ClHH}$ prevented $\mathrm{Cl}$-AKI group were still higher than the baseline values, their elevation was significantly reduced, and blood pressure and heart rate were significantly lower than those in the $\mathrm{Cl}$ - AKI group, while the renal pathological injury was significantly reduced. These suggest that $\mathrm{ClHH}$ has a protective effect against contrast-induced acute kidney injury.

ROS plays an important role in the development of contrast nephropathy, which can cause direct damage to vascular endothelial cells and renal tubules, and in turn aggravates the hypoxic damage to renal parenchyma caused by vascular endothelial dysfunction and dysregulation of renal tubular transport[6]. SOD is an enzyme that can restore the balance of cellular redox disorders caused by local ischemia and hypoxia, and SOD catalyzes the disproportionation of $\mathrm{O}_{2}$ radicals in $\mathrm{H}_{2} \mathrm{O}_{2}$, which can prevent the accumulation of reactive oxygen species and play a role in scavenging oxygen free radicals[29]. In the kidney tissue of contrast nephropathy, ROS is increased, SOD activity is decreased, and MDA levels are increased[23,30]. Several studies have shown that increased SOD activity attenuates tissue or cellular damage caused by oxidative stress[31-34]. In the present study, renal SOD activity was significantly lower and MDA levels were significantly higher in the Cl-AKI group, which is consistent with other related studies. Renal SOD activity was significantly higher and MDA levels were lower in the $\mathrm{CIHH}$ prevented $\mathrm{Cl}-$ $\mathrm{AKI}$ group than in the $\mathrm{Cl}-\mathrm{AKI}$ group, indicating that $\mathrm{ClHH}$ pretreatment could protect kidney by attenuating renal oxidative stress.

Although the pathogenesis of $\mathrm{Cl}-\mathrm{AKI}$ remains unclear, ischemia and hypoxia play an important role in the development and progression of contrast nephropathy[2]. More than 100 genes have been identified to cause a significant increase in HIF-1a in response to renal hypoxia[35]. By upregulating of renal HIF-1a levels could ameliorate renal fibrosis and glomerular mesangial hyperplasia in diabetic rats[10]. Recent studies have shown that activation of the HIF-1a/BNIP3 signaling pathway under hypoxic conditions results in enhanced mitochondrial autophagy, which can have a protective effect on tissues or cells. Lin et al. showed that renal injury and apoptosis were exacerbated in BNIP3 knockout mice given contrast agents and in HK2 cells transfected in advance with si-BNIP3 to silence BNIP3[8].In a study by Fu et al, inhibition of mitochondrial autophagy in the kidneys of HIF-1a knockout ischemia/reperfusion mice exacerbated tubular apoptosis and renal injury, and increased BNIP3 expression in the kidneys of knockout mice resulted in enhanced mitochondrial autophagy, reduced tubular apoptosis and renal injury, indicating that the HIF-1a/BNIP3 signaling pathway mediates ischemia/reperfusion injury and that activation of this pathway may protect against ischemia/reperfusion injury[7].In the present study, the renal HIF-1 $a$ and BNIP3 protein expression levels were increased in the Cl-AKI group compared to CTL 
group, and $\mathrm{ClHH}$ prevented $\mathrm{Cl}-\mathrm{AKI}$ group were significantly higher compared to $\mathrm{Cl}-\mathrm{AKI}$ group, indicating that $\mathrm{ClHH}$ preadaptation could enhance mitochondrial autophagy through activation of the HIF-1a/BNIP3 signaling pathway to protect against contrast-induced renal injury.

Not only the toxic effects of the contrast agent itself, but also ischemia and hypoxia, oxidative stress injury and apoptosis play an important role in the development and progression of Cl-AKI. Several studies have shown that caspase 3 activity increases and the level of anti-apoptotic factor BCL-2 decreases in acute kidney injury, leading to increased apoptosis, which caused renal tubular epithelial cell injury, and that administration of drugs to inhibit aspase 3 activity can reduce cell apoptosis and thus improve kidney injury[25, 36-38].Caspase3 was inactive, but its activated product, cleaved-caspase3,which could activate PARP caused apoptosis. Romano et al. showed that caspase3 and PARP levels were reduced and apoptosis was increased in a mouse model administered with contrast agents suggesting that caspase3-dependent apoptotic pathway mediates iodine contrast-induced acute kidney injury[39].In the present experiment, caspase 3 and PARP levels in the kidney tissues of rats in the Cl-AKI group were significantly lower compared to CTL group, but the levels of caspase 3 and PARP in the CIHH prevented $\mathrm{Cl}-\mathrm{AKI}$ group were significantly increased compared to $\mathrm{Cl}-\mathrm{AKI}$ group, indicating that $\mathrm{ClHH}$ pretreatment played a protective role against contrast-induced acute kidney injury by increasing the anti-apoptotic capacity of renal cells.

\section{Conclusions}

The present study showed that $\mathrm{ClHH}$ pretreatment could weaken renal injury in $\mathrm{Cl}-\mathrm{AKI}$ rats, and these beneficial effects were mainly achieved by modulating HIF-1a/BNIP3 signaling pathway, anti-apoptosis, and anti-oxidative stress. Therefore, $\mathrm{ClHH}$ pretreatment may be a potential approach to prevent $\mathrm{Cl}-\mathrm{AKI}$. The shortcoming of the present experiment is that in vitro studies of renal cells were not performed.

\section{Declarations}

Competing Interests:

No conflicts of interest, financial or otherwise, are declared by the author(s).

Authors' Contributions:

Kai-min Yin made a major contribution to this paper.

Acknowledgements:

We thank Prof. Hui-jie Ma and his group in the Physiology Department of Hebei Medical University for providing the Hypobaric hypoxic chamber for this study and for their help in the experiments.

Data availability statement: 
The data sets generated and / or analyzed during this study are not public due to the data store in Baidu online disk, but Baidu online disk is not a public network in the world, not everyone can relevant information links can be obtained, but can be obtained from the corresponding author upon reasonable request.

Original data owner declaration:

Each person who needs this data can obtain relevant data from the author Kai-min Yin and contact the email 854443280@qq.com.

\section{Reference}

1. F?hling, M., et al., Understanding and preventing contrast-induced acute kidney injury. Nat Rev Nephrol, 2017. 13(3): p. 169-180.

2. Katzberg, R.W., Contrast medium-induced nephrotoxicity: which pathway? Radiology, 2005. 235(3): p. 752-5.

3. Topalo?lu, U.S., et al., Effects of thymoquinone in prevention of experimental contrast-induced nephropathy in rats. Iran J Basic Med Sci, 2019. 22(12): p. 1432-1439.

4. Lee, P., N.S. Chandel, and M.C. Simon, Cellular adaptation to hypoxia through hypoxia inducible factors and beyond. Nat Rev Mol Cell Biol, 2020. 21(5): p. 268-283.

5. Gonzalez, F.J., C. Xie, and C. Jiang, The role of hypoxia-inducible factors in metabolic diseases. Nat Rev Endocrinol, 2018. 15(1): p. 21-32.

6. Yang, K.J., et al., Inhibition of xanthine oxidoreductase protects against contrast-induced renal tubular injury by activ ating adenosine monophosphate-activated protein kinase. Free Radic Biol Med, 2019. 145: p. 209-220.

7. Fu, Z.J., et al., HIF-1|Á-BNIP3-mediated mitophagy in tubular cells protects against renal ischemia/reperfusion injury. Redox Biol, 2020. 36: p. 101671.

8. Lin, Q., et al., Inhibiting NLRP3 inflammasome attenuates apoptosis in contrast-induced acute kidney injury through th e upregulation of HIF1A and BNIP3-mediated mitophagy. Autophagy, 2021. 17(10): p. 2975-2990.

9. Romano, G., et al., Contrast agents and renal cell apoptosis. Eur Heart J, 2008. 29(20): p. 2569-76.

10. Tian, Y.M., et al., Chronic intermittent hypobaric hypoxia ameliorates diabetic nephropathy through enhancing HIF1 signal ing in rats. Diabetes Res Clin Pract, 2016. 118: p. 90-7.

11. Sanchis-Gomar, F., J. Vi?a, and G. Lippi, Intermittent hypobaric hypoxia applicability in myocardial infarction prevention and recovery. J Cell Mol Med, 2012. 16(5): p. 1150-4.

12. Li, N., et al., Chronic Intermittent Hypobaric Hypoxia Ameliorates Renal Vascular Hypertension Through Up-regulating NOS in Nucleus Tractus Solitarii. Neurosci Bull, 2019. 35(1): p. 79-90.

13. Cui, F., et al., Chronic intermittent hypobaric hypoxia protects vascular endothelium by ameliorating autophagy in met abolic syndrome rats. Life Sci, 2018. 205: p. 145-154. 
14. Yu, B., et al., ClHH protects the heart against left ventricular remodelling and myocardial fibrosis by balancing the renin-angiotensin system in SHR. Life Sci, 2021. 278: p. 119540.

15. Li, J., et al., Preservation of TSPO by chronic intermittent hypobaric hypoxia confers antiarrhythmic activity. J Cell Mol Med, 2011. 15(1): p. 134-40.

16. Kohutova, J., et al., Anti-arrhythmic Cardiac Phenotype Elicited by Chronic Intermittent Hypoxia Is Associated With Alterat ions in Connexin-43 Expression, Phosphorylation, and Distribution. Front Endocrinol (Lausanne), 2018. 9: p. 789.

17. Aguilar, M., et al., Mechanisms of Cardiovascular Protection Associated with Intermittent Hypobaric Hypoxia Exposure in a Rat Model: Role of Oxidative Stress. Int J Mol Sci, 2018. 19(2).

18. Li, X., et al., Enhancement of Glucose Metabolism via PGC-1;Á Participates in the Cardioprotection of Chronic Intermit tent Hypobaric Hypoxia. Front Physiol, 2016. 7: p. 219.

19. Yuan, F., et al., Chronic Intermittent Hypobaric Hypoxia Improves Cardiac Function through Inhibition of Endoplasmic Re ticulum Stress. Sci Rep, 2017. 7(1): p. 7922.

20. Cheng, W.J., et al., Chronic intermittent hypobaric hypoxia attenuates skeletal muscle ischemiareperfusion injury in mice. Life Sci, 2019. 231: p. 116533.

21. Cui, F., et al., The Effect of Autophagy on Chronic Intermittent Hypobaric Hypoxia Ameliorating Liver Damage in Metabo lic Syndrome Rats. Front Physiol, 2020. 11: p. 13.

22. Bae, E., et al., Paricalcitol Attenuates Contrast-Induced Acute Kidney Injury by Regulating Mitophagy and Senescence. Oxid Med Cell Longev, 2020. 2020: p. 7627934.

23. Zhao, Z., et al., Sulforaphane Attenuates Contrast-Induced Nephropathy in Rats via Nrf2/HO-1 Pathway. Oxid Med Cell Longev, 2016. 2016: p. 9825623.

24. Bartorelli, A.L. and G. Marenzi, High Urine Output With Matched Hydration?for Cl-AKI Prevention: "Salus Per Aquam" (Health Through Wat er). JACC Cardiovasc Interv, 2017. 10(4): p. 364-366.

25. Wang, F., et al., Magnolin protects against contrast-induced nephropathy in rats via antioxidation and antiapoptosis. Oxid Med Cell Longev, 2014. 2014: p. 203458.

26. Birck, R., et al., Acetylcysteine for prevention of contrast nephropathy: meta-analysis. Lancet, 2003. 362(9384): p. 598-603.

27. Komiyama, K., et al., Sodium Bicarbonate-Ascorbic Acid Combination for Prevention of ContrastInduced Nephropathy in Chroni c Kidney Disease Patients Undergoing Catheterization. Circ J, 2017. 81(2): p. 235-240.

28. Marenzi, G., et al., Prevention of contrast nephropathy by furosemide with matched hydration: the MYTHOS (Induced Diuresis With Matched Hydration Compared to Standard Hydration for Contrast Induced Nephropathy Prevention) trial. JACC Cardiovasc Interv, 2012. 5(1): p. 90-7.

29. Fridovich, I., Superoxide dismutases. Annu Rev Biochem, 1975. 44: p. 147-59.

30. Kurtoglu, T., et al., Ozone preconditioning attenuates contrast-induced nephropathy in rats. J Surg Res, 2015. 195(2): p. 604-11. 
31. Li, X., et al., Mechanisms of Protective Effect of Ramulus Mori Polysaccharides on Renal Injury in High-Fat Diet/Stre ptozotocin-Induced Diabetic Rats. Cell Physiol Biochem, 2015. 37(6): p. 2125-34.

32. Xu, W., et al., TXNIP mediated the oxidative stress response in glomerular mesangial cells partially through AMPK pathway. Biomed Pharmacother, 2018. 107: p. 785-792.

33. Ebaid, H., et al., Folic acid and melatonin mitigate diabetic nephropathy in rats via inhibition of oxidative stress. Nutr Metab (Lond), 2020. 17: p. 6.

34. Zhu, X., et al., Islet Transplantation Attenuating Testicular Injury in Type 1 Diabetic Rats Is Associated with Suppression of Oxidative Stress and Inflammation via Nrf-2/HO-1 and NF-KB Pathways. J Diabetes Res, 2019. 2019: p. 8712492.

35. Yang, Z.Z., et al., Redox regulation of HIF-1alpha levels and HO-1 expression in renal medullary interstitial cells. Am J Physiol Renal Physiol, 2003. 284(6): p. F1207-15.

36. Solomon, R. and H.L. Dauerman, Contrast-induced acute kidney injury. Circulation, 2010. 122(23): p. 2451-5.

37. Wang, Z., et al., GSK3beta promotes apoptosis after renal ischemic injury. J Am Soc Nephrol, 2010. 21(2): p. 284-94.

38. Deng, J., et al., Rosuvastatin attenuates contrast-induced nephropathy through modulation of nitric oxide, inflammatory responses, oxidative stress and apoptosis in diabetic male rats. J Transl Med, 2015. 13: p. 53.

39. Khaleel, S.A., et al., Lansoprazole halts contrast induced nephropathy through activation of Nrf2 pathway in rats. Chem Biol Interact, 2017. 270: p. 33-40.

\section{Figures}




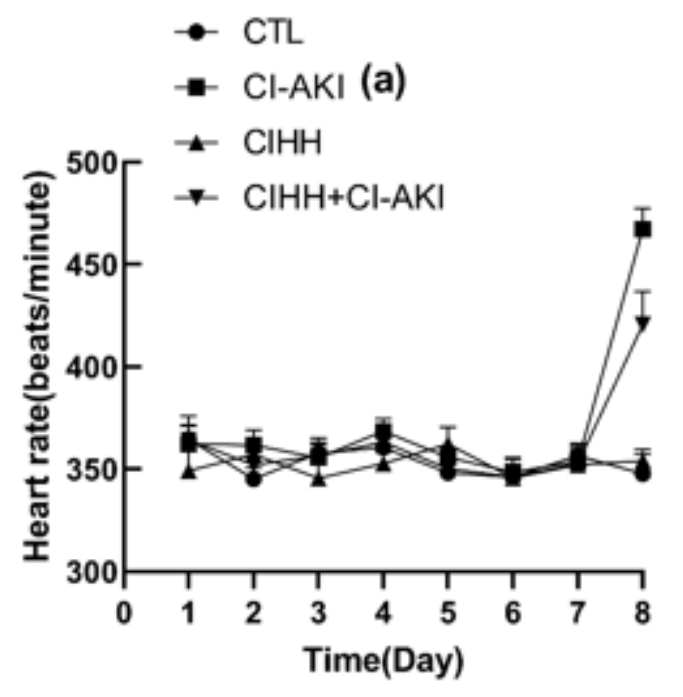

(b)
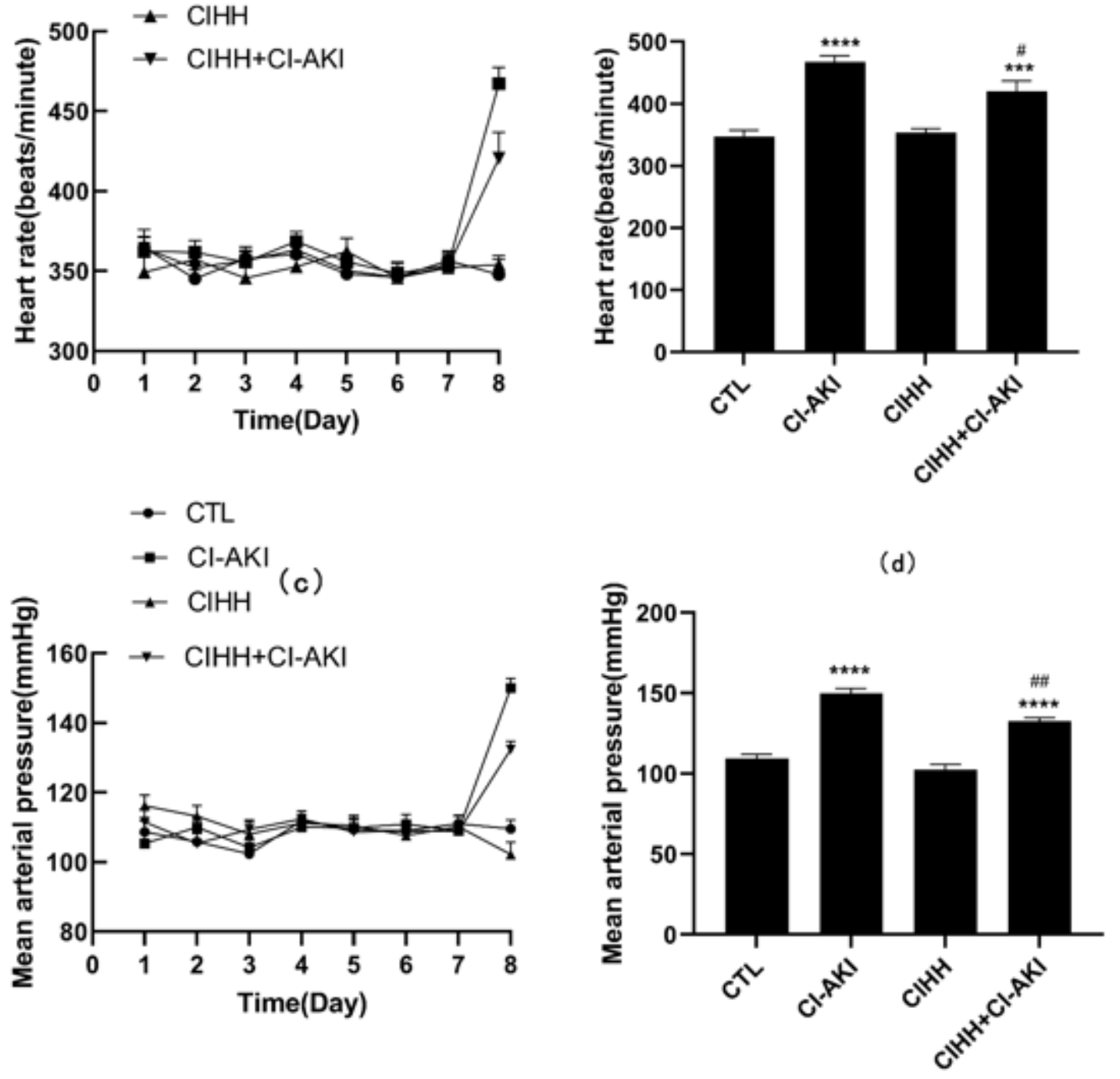

Figure 1

Effects of $\mathrm{ClHH}$ on heart rate and mean arterial pressure. The heart rate and mean arterial pressure dates were collected from seven days before injection to 24 hours after injection. The heart rate dates from one day to seven days before injection and 24 hours after injection(a), the heart rate dates at 24 hours after injection (b). The mean arterial pressure dates from one day to seven days before injection and 24 hours after injection (c), the mean arterial pressure dates at 24 hours after injection (d). CTL, normal-controlled group; $\mathrm{Cl}-\mathrm{AKI}$, contrast induced acute kidney injury group; $\mathrm{ClHH}$, chronic intermittent hypobaric hypoxia pretreatment group; $\mathrm{ClHH}+\mathrm{Cl}-\mathrm{AKI}$, contrast induced acute kidney injury group with chronic intermittent hypobaric hypoxia pretreatment. Data were presented as means \pm SEM $(n=6) .{ }^{* \star * P} P<0.001$, $\star \star \star \star *<0.0001$ versus. CTL; $\# p<0.05, \# \# p<0.01$ versus. Cl-AKI. 
(a)

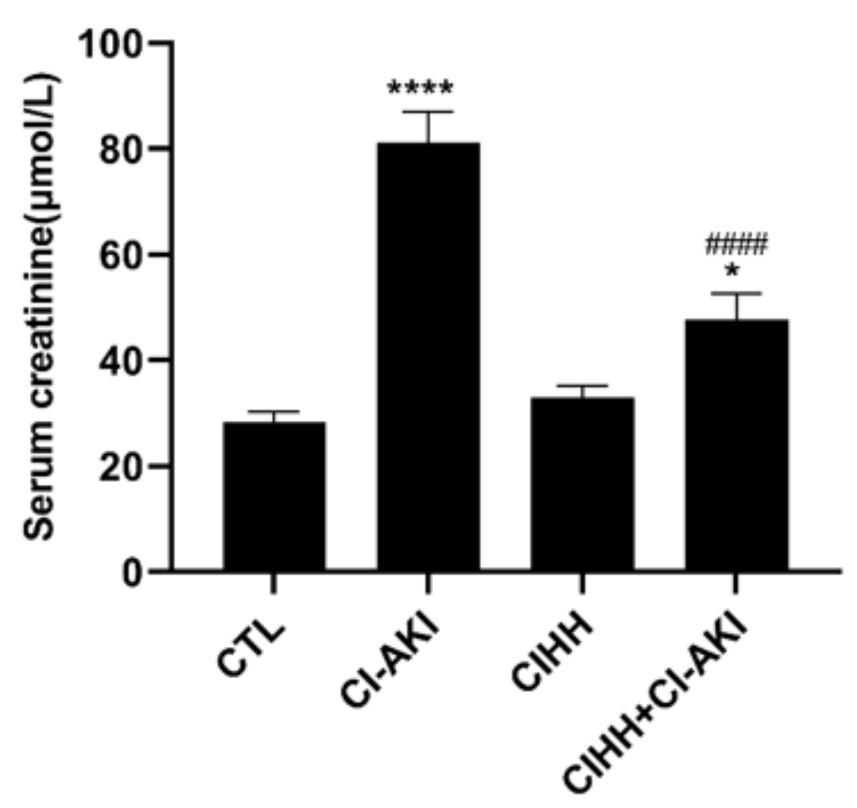

(b)

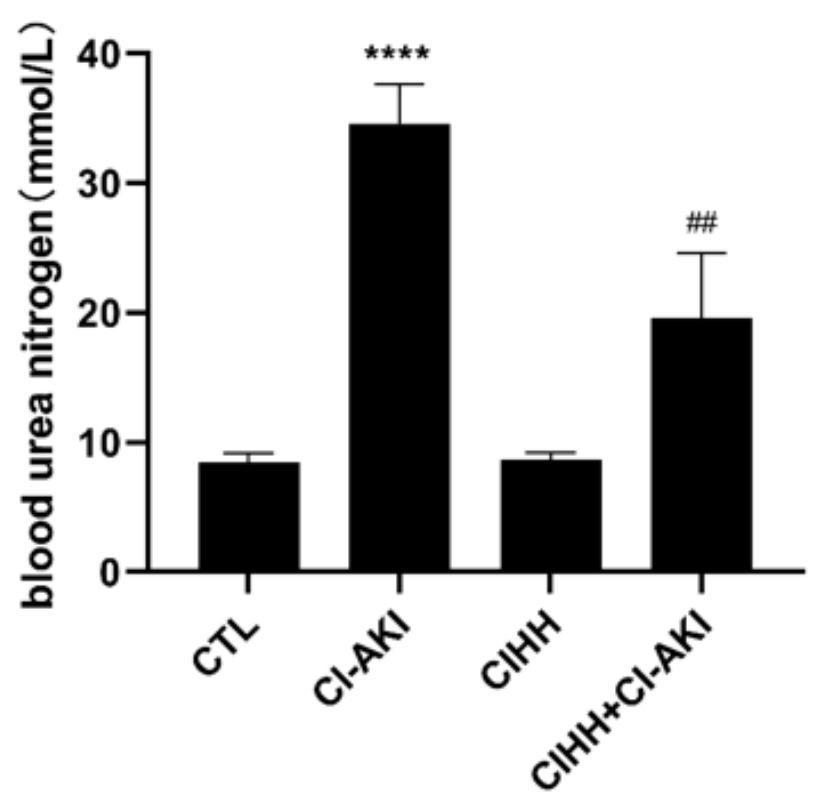

Figure 2

Effects of $\mathrm{ClHH}$ on renal function. Blood samples were collected through caudal vein before and 24 hours after contrast medium injection. Serum creatinine (a), blood nitrogen urea (b). CTL, normal-controlled group; $\mathrm{Cl}-\mathrm{AKI}$, contrast induced acute kidney injury group; $\mathrm{ClHH}$, chronic intermittent hypobaric hypoxia pretreatment group; $\mathrm{ClHH}+\mathrm{Cl}-\mathrm{AKI}$, contrast induced acute kidney injury group with chronic intermittent hypobaric hypoxia pretreatment. Data were presented as means \pm SEM $(n=6) .{ }^{*} P<0.05,{ }^{\star \star \star *} P P<0.0001$ versus CTL; \#\#p<0.01, \#\#\#\#p<0.0001versusCI-AKI. 


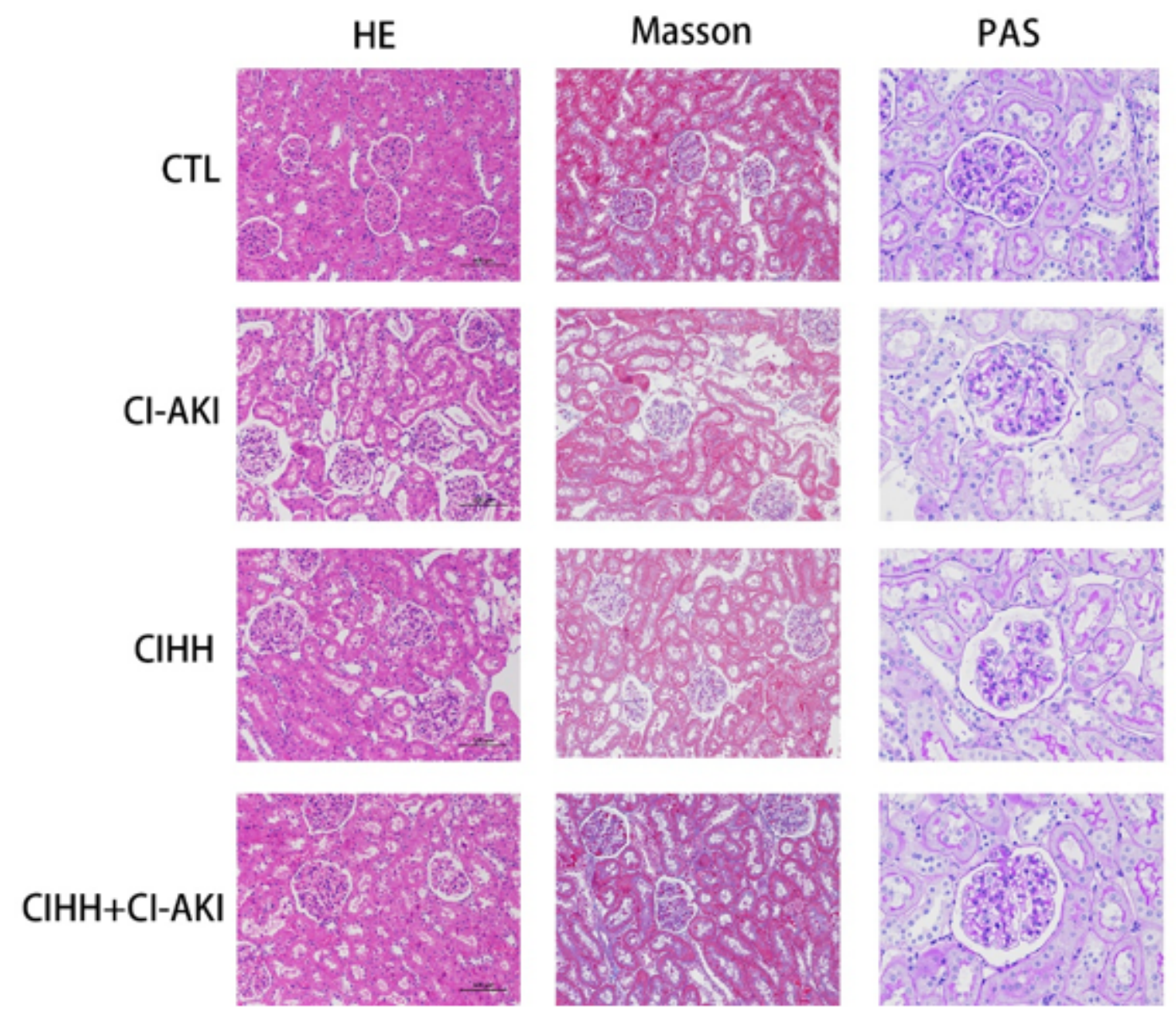

\section{Figure 3}

Effects of $\mathrm{ClHH}$ on renal histological examination. $\mathrm{HE}$, hematoxylin eosin staining; PAS, periodic acid Schiff reaction; Masson, Masson trichrome. CTL, normal-controlled group; $\mathrm{Cl}-\mathrm{AKI}$, contrast induced acute kidney injury group; $\mathrm{ClHH}$, chronic intermittent hypobaric hypoxia pretreatment group; $\mathrm{ClHH}+\mathrm{Cl}-\mathrm{AKI}$, contrast induced acute kidney injury group with chronic intermittent hypobaric hypoxia pretreatment. 
(a)

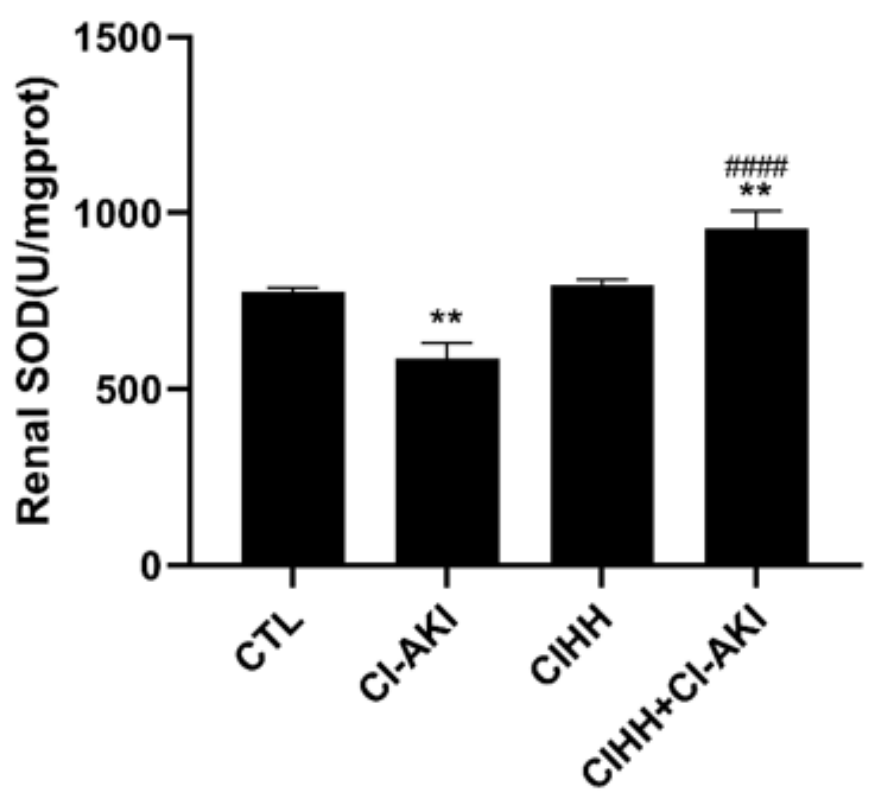

(b)

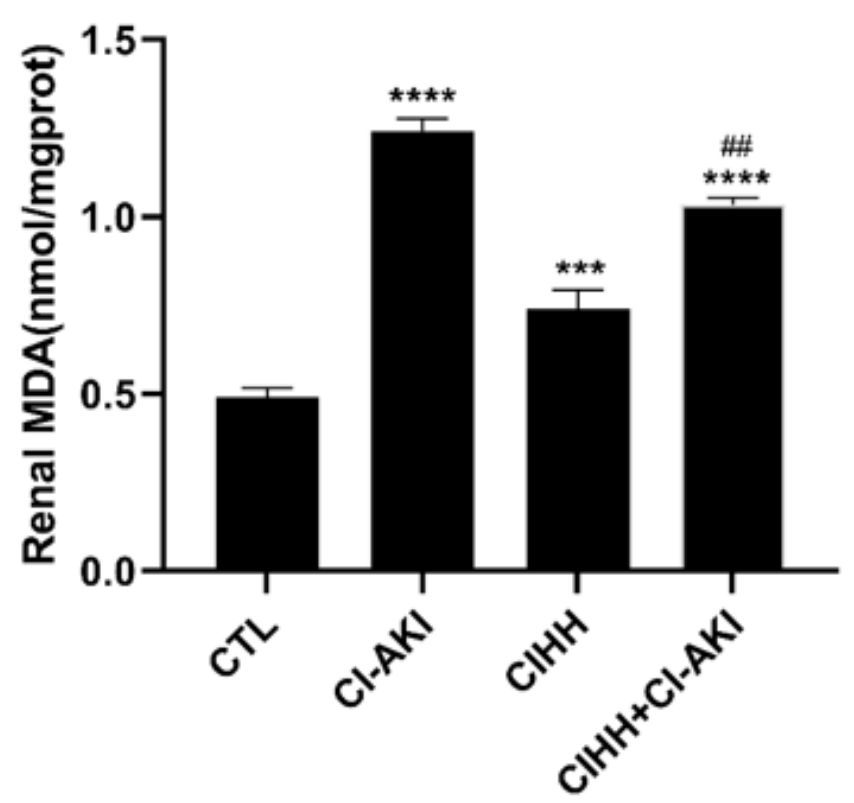

Figure 4

Effects of $\mathrm{ClHH}$ on renal oxidative stress. The levels of malondialdehyde (MDA) and superoxide dismutase (SOD) were measured to determine the alteration of oxidative stress. Renal MDA(a), Renal SOD (b). CTL, normal-controlled group; Cl-AKI, contrast induced acute kidney injury group; $\mathrm{ClHH}$, chronic intermittent hypobaric hypoxia pretreatment group; $\mathrm{ClHH}+\mathrm{Cl}-\mathrm{AKI}$, contrast induced acute kidney injury group with chronic intermittent hypobaric hypoxia pretreatment. Dates were presented as means \pm SEM

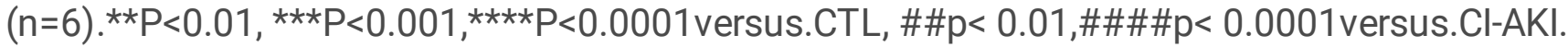




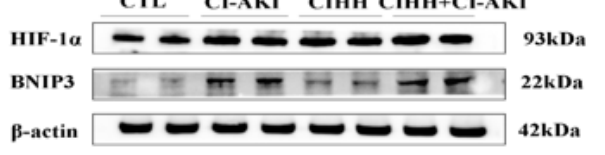

b

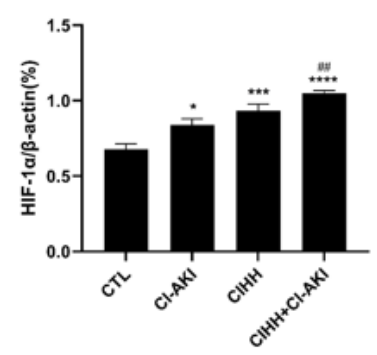

c

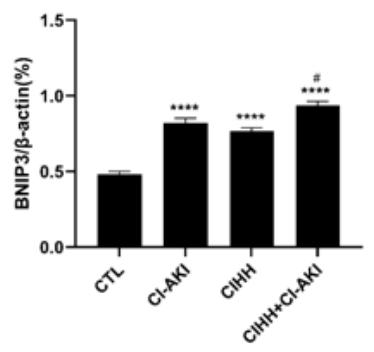

d

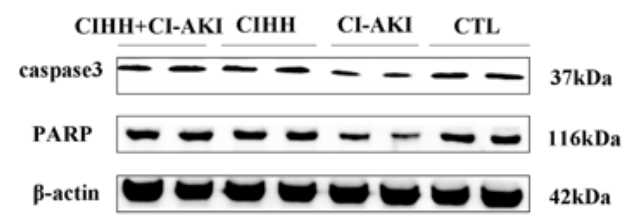

e

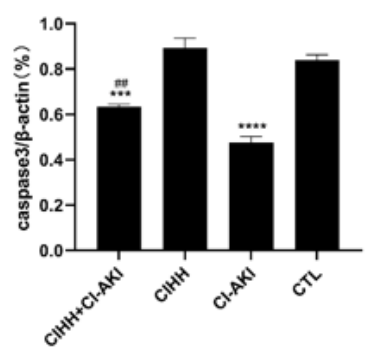

f

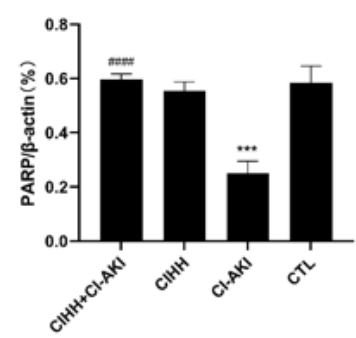

\section{Figure 5}

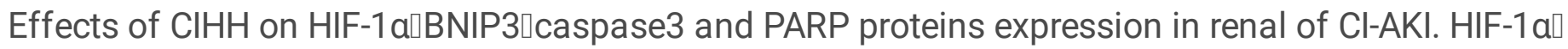
BNIP3 $₫$-actin in the picture a from the same gel. Caspase3ロPARP $\beta$-actin in the picture $d$ from the same

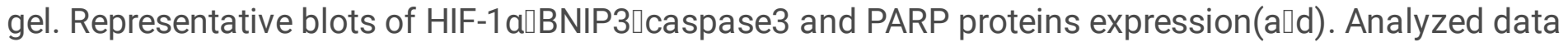

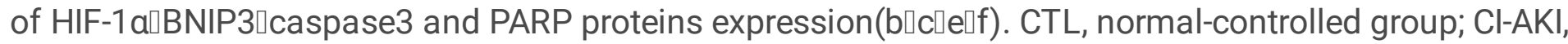
contrast induced acute kidney injury group; $\mathrm{ClHH}$, chronic intermittent hypobaric hypoxia pretreatment 
group; $\mathrm{ClHH}+\mathrm{Cl}-\mathrm{AKI}$, contrast induced acute kidney injury group with chronic intermittent hypobaric hypoxia pretreatment. Dates were presented as means \pm SEM $(n=6) .{ }^{*} P<0.05$, ${ }^{\star \star \star} P<0.001$, ${ }^{\star \star \star \star} P<0.0001$ versus.CTL; $\# p<0.05$,\#\#p<0.01,\#\#\#\#p<0.0001versus. Cl-AKI. The uncropped blots are presented in Supplementary Figure. 